\title{
Acoustic pulse propagation in an urban environment using a three-dimensional numerical simulation
}

\author{
Ravish Mehra ${ }^{\text {a) }}$ \\ Department of Computer Science, University of North Carolina at Chapel Hill, Chapel Hill, \\ North Carolina 27599-3175 \\ Nikunj Raghuvanshi \\ Microsoft Research, One Microsoft Way, Redmond, Washington 98052 \\ Anish Chandak \\ Department of Computer Science, University of North Carolina at Chapel Hill, Chapel Hill, \\ North Carolina 27599-3175 \\ Donald G. Albert and D. Keith Wilson \\ U.S. Army Cold Regions Research and Engineering Laboratory, 72 Lyme Road, Hanover, \\ New Hampshire 03755-1290 \\ Dinesh Manocha \\ Department of Computer Science, University of North Carolina at Chapel Hill, Chapel Hill, \\ North Carolina 27599-3175
}

(Received 29 July 2013; revised 20 March 2014; accepted 10 April 2014)

\begin{abstract}
Acoustic pulse propagation in outdoor urban environments is a physically complex phenomenon due to the predominance of reflection, diffraction, and scattering. This is especially true in non-line-of-sight cases, where edge diffraction and high-order scattering are major components of acoustic energy transport. Past work by Albert and Liu [J. Acoust. Soc. Am. 127, 1335-1346 (2010)] has shown that many of these effects can be captured using a two-dimensional finite-difference time-domain method, which was compared to the measured data recorded in an army training village. In this paper, a full three-dimensional analysis of acoustic pulse propagation is presented. This analysis is enabled by the adaptive rectangular decomposition method by Raghuvanshi, Narain and Lin [IEEE Trans. Visual. Comput. Graphics 15, 789-801 (2009)], which models sound propagation in the same scene in three dimensions. The simulation is run at a much higher usable bandwidth (nearly $450 \mathrm{~Hz}$ ) and took only a few minutes on a desktop computer. It is shown that a three-dimensional solution provides better agreement with measured data than two-dimensional modeling, especially in cases where propagation over rooftops is important. In general, the predicted acoustic responses match well with measured results for the source/sensor locations. [http://dx.doi.org/10.1121/1.4874495]
\end{abstract}

PACS number(s): 43.28.Js, 43.28.En [PBB]

Pages: $3231-3242$

\section{INTRODUCTION}

Acoustic propagation in urban environments is a physically complex problem that has many practical applications. In urban planning and city design, acoustic propagation models can inform decisions on the location of noisesensitive buildings like hospitals and schools. ${ }^{1}$ Accurate computational modeling is also useful in designing baffles near areas of high traffic to control noise levels in residential neighborhoods. ${ }^{2,3}$ Acoustic modeling is also useful in soundsource localization: Numerous sensors are placed in an urban environment to detect sound events and calculate the sound source's position using the peak arrival times of the sound waves. This computation of the sound source's position can be used for gunshot localization, which is useful for crime control in urban areas ${ }^{4}$ and in many military applications. ${ }^{5}$

Acoustic propagation modeling for urban areas is a challenging computational problem because of the complex

\footnotetext{
a) Author to whom correspondence should be addressed. Electronic mail: ravish.mehra07@gmail.com
}

building geometry and large domain size. High-order diffraction and scattering play a significant role in acoustic energy transport in urban areas, especially in cases when the source and receiver are not in line-of-sight. Previous work in the field has mainly focused on continuous noise sources to determine statistical quantities like reverberation time and noise levels. ${ }^{6-8}$ However, these are gross acoustic parameters and do not give a detailed view of the actual propagation. Geometric techniques have been used to evaluate noise levels and calculate sound propagation in urban streets. ${ }^{9,10}$ However, due to these techniques' inherent assumption of rectilinear propagation of sound waves, modeling wave effects such as diffraction and interference remains a significant challenge with these techniques.

Recent work in numerical techniques has focused on the use of acoustic pulse propagation techniques in the time domain to get detailed characteristics of the complex propagation effects in urban scenes. Time-domain pulse propagation is preferred in urban acoustic modeling as it gives direct insight into the propagation by producing animations of pressure wavefronts. This allows one to quickly inspect the propagation path corresponding to dominant peaks in the 
response at a given sensor location. Recent studies used a finite-difference time-domain simulation to model acoustic pulse propagation and compared the results with real-world measurements. ${ }^{11-13}$ Those studies were limited to 2D modeling due to the high computational cost and memory requirement of the finite-difference technique for this large domain size.

In this paper, a full 3D analysis of acoustic pulse propagation in the time domain is presented. Our analysis is made on a virtual $3 \mathrm{D}$ model of the same scene as the prior $2 \mathrm{D}$ investigation; ${ }^{13}$ this $3 \mathrm{D}$ analysis is made computationally feasible by using Adaptive Rectangular Decomposition (ARD), ${ }^{14}$ an efficient time-domain numerical solver, which allows us to model propagation in this scene in three dimensions. ARD is more computation- and memory-efficient for homogenous media than the finite-difference time-domain technique. The improved efficiency allows the ARD simulations to have a much higher usable bandwidth (up to midrange frequencies of $450 \mathrm{~Hz}$, compared to $200 \mathrm{~Hz}$ in prior work $^{13}$ ), while taking just a few minutes on a desktop computer. A detailed analysis of errors between measured data and simulated data is performed, showing that 3D simulations provide better agreement with measured data than $2 \mathrm{D}$ simulations. The agreement is markedly better in cases where propagation over rooftops is important, a case which the 2D modeling cannot capture at all. In general, the predicted acoustic responses match well with measured results for most source/sensor locations, with typical errors being on the order of $3 \mathrm{~dB}$. Visualizations of the time-domain simulation show that a rooftop-diffracted path provides important energy contributions at certain locations in the scene.

\section{PREVIOUS WORK}

Over the years, many techniques have been developed to study acoustic propagation in urban environments. ${ }^{1}$ Analytical solutions are available for simple scenarios involving building edges and noise barriers. ${ }^{15}$ Theoretical predictions have been used to predict the noise levels in urban street complexes. ${ }^{16}$ Statistical analysis ${ }^{17,18}$ has been performed on measured data to analyze the reverberation time and sound levels in streets and to study the relationship between different noise descriptors in urban areas. Many ray-tracing based approaches ${ }^{8,9}$ have been proposed to evaluate the increase in traffic noise for street canyons due to the presence of buildings. Kang et al. ${ }^{10}$ used a radiosity-based model to calculate sound propagation in interconnected urban streets. And the radiosity-based model has been combined with the imagesource method to handle diffuse and geometrical boundaries for street canyon scenarios. ${ }^{19}$

Typical numerical approaches used to study urban acoustic propagation are Finite Difference Time-Domain, ${ }^{20}$ Finite Element Method, ${ }^{21}$ Boundary Element Method, ${ }^{22}$ Equivalent Source Method, ${ }^{23}$ and Pseudo-Spectral TimeDomain. ${ }^{24}$ The boundary element method has been applied to acoustic propagation in areas with noise barriers ${ }^{25}$ and in outdoor scenes. ${ }^{26}$ To model sound propagation in city canyons, Ogren and Kropp (2004) used the equivalent source method, and Van Renterghem et al. (2006) used a coupled finite-difference parabolic equation method. ${ }^{27,28}$ Ovenden et al. (2009) coupled the analytical calculation to a parabolic equation method for modeling noise propagation in urban freeways. ${ }^{29}$ To model atmospheric sound propagation, a pseudo-spectral time-domain (PSTD) approach $^{30}$ was proposed. The finite-difference approach has been used in recent years to model acoustic pulse propagation in urban environments, and the results were compared with measured waveforms recorded at the physical site, including propagation for a right-angled wall, ${ }^{11}$ a single building, ${ }^{12}$ and a training village with multiple buildings. ${ }^{13}$ However, due to computational limitations, all these approaches have been limited to propagation in two dimensions.

Some recent studies have modeled three-dimensional sound propagation. Ketcham et al. (2008) used a finitedifference approach for modeling the effect of urban infrastructure on sound scattering in three dimensions, ${ }^{31}$ but the modeling required a computer cluster with hundreds of processors. Pollès et al. (2004) proposed a diffusion-equationbased approach to model 3D sound propagation in urban areas with multiple buildings. ${ }^{32}$ Recently, a fast and efficient time-domain approach was proposed; this technique, called adaptive rectangular decomposition, solves the wave equation in three dimensions for spatially invariant speed of sound. ${ }^{14,33}$ It is related to the PSTD technique but avoids the discrete integration in time by using analytical solutions of the wave equation for rectangular domains. For a more detailed survey of outdoor sound propagation techniques, the survey paper $^{34}$ is recommended.

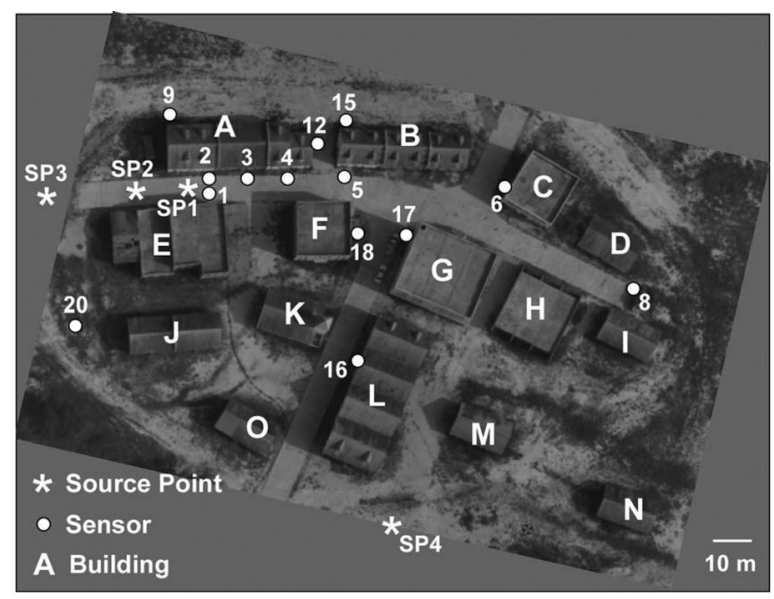

(a)

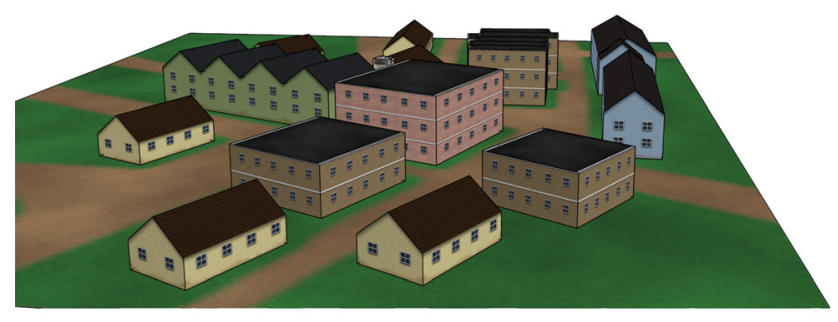

(b)

FIG. 1. (Color online) (a) Top view of the urban scene used in the experimental study. Reproduced with permission from Albert and Liu (2010). (b) An approximate 3D model of the scene constructed based on the 2D layout, photographs of the scene, and heights of the buildings' corners and roof tops. 


\section{MEASUREMENTS}

In this section, we discuss the real-world measurements used in the validation of the numerical simulation. This dataset was presented in the work of Albert and Liu (2010). ${ }^{13}$ We provide a brief discussion here, but more details can be found in their previous work.

\section{A. Scene layout}

The experiment was conducted in an artificial village spanning a $150 \mathrm{~m} \times 150 \mathrm{~m}$ area with 15 buildings and two cross-streets: "Main street" running perpendicular to the "Church street." Figure 1(a) shows the 2D layout (top view) of the urban scene. The buildings in the village were two or three stories tall and made up of concrete blocks. The ground areas consisted of streets, grass areas, and hard-packed soil.

\section{B. Weather conditions}

The experiment was conducted over two sunny days with temperature, wind, and relative humidity variation between $8^{\circ} \mathrm{C}$ to $19^{\circ} \mathrm{C}, 2$ to $5 \mathrm{~m} / \mathrm{s}$, and $30 \%-50 \%$, respectively.

\section{Sources}

Acoustic pulses were produced by using small explosives of $0.57 \mathrm{~kg}$ of $\mathrm{C} 4$ suspended at a height of $1.5 \mathrm{~m}$ from the ground. The measurements were recorded for four source positions, SP1-SP4.

\section{Receivers}

Sensors were placed at 14 different receiver positions spread throughout the scene, in both line-of-sight (LOS) and non-line-of-sight (NLOS) positions. These sensors were connected to digital seismographs that recorded the pressure signal at a sampling rate of 5 or $8 \mathrm{kHz}$.

\section{ADAPTIVE RECTANGULAR DECOMPOSITION NUMERICAL MODELING}

In this section, we give an overview of the adaptive rectangular decomposition (ARD) simulation technique for modeling acoustic pulse propagation. ${ }^{14,33,35}$

\section{A. The adaptive rectangular decomposition method}

Our starting point is the wave equation for constant sound speed,

$$
\frac{\partial^{2} p}{\partial t^{2}}-c^{2} \nabla^{2} p=f(\mathbf{x}, t),
$$

where $p(\mathbf{x}, t)$ is the time-varying pressure field, $f(\mathbf{x}, t)$ is the force term corresponding to the volume sound sources, $c$ is the speed of sound, and $\nabla^{2}$ is the Laplacian operator. The speed of sound in the medium is assumed to be spatially invariant.

Figure 2 demonstrates the main stages of the ARD pipeline. The technique starts with a 3D model of the scene, voxelizes the air volume, and then decomposes the voxelization into rectangular partitions. The wave equation has a known analytical solution for rectangular domains for spatially

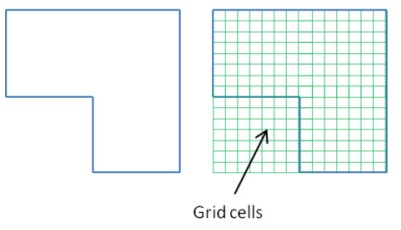

Domain

Voxelization

$$
\text { (a)Preprocessing }
$$

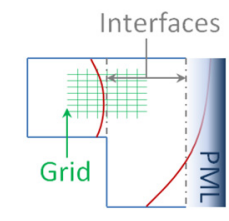

Current Field (t)

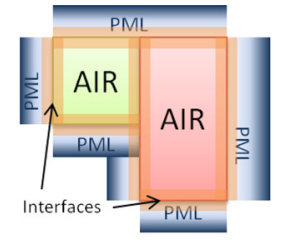

Rectangular

Decomposition

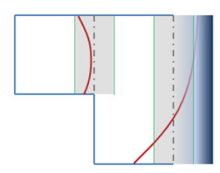

Interface handling and DCT

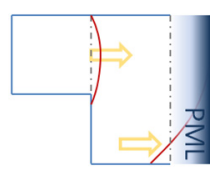

Mode Update and IDCT $(\mathrm{t}+\mathrm{dt})$
(b)Simulation

FIG. 2. (Color online) Different stages of the Adaptive Rectangular Decomposition simulator.

invariant speed of sound. Consider a rectangle in 3D of size $\left(l_{x}, l_{y}, l_{z}\right)$ with perfectly reflecting walls. The analytical solution of the wave equation in this case can be written as

$$
p(\mathbf{x}, t)=\sum_{i=\left(i_{x}, i_{y}, i_{z}\right)} m_{i}(t) \Phi_{i}(\mathbf{x}),
$$

where $i_{x}$ are $\mathrm{x}$-indices in the range $\left[1-l_{x}\right]$ and, $i_{y}$ and $i_{z}$ are $\mathrm{y}$ - and z-indices, respectively. Here $i=\left(i_{x}, i_{y}, i_{z}\right)$ is a generalized index over three dimensions, $m_{i}(t)$ are time-varying mode coefficients, and $\Phi_{i}(\mathbf{x})$ are eigenfunctions of the Laplacian, given by

$$
\Phi_{i}(\mathbf{x})=\cos \left(\frac{\Pi i_{x}}{l_{x}} x\right) \cos \left(\frac{\Pi i_{y}}{l_{y}} y\right) \cos \left(\frac{\Pi i_{z}}{l_{z}} z\right),
$$

for a perfectly reflecting boundary condition. In discrete interpretation, pressure can be transformed into mode coefficients $M_{i}$ at each time step by a Discrete Cosine Transform (DCT), since the eigenfunctions are cosines. The update rule for mode coefficients can be derived by taking a DCT of the wave equation and solving the resultant simple harmonic oscillator system, giving

$$
M_{i}^{n+1}=2 M_{i}^{n} \cos \left(w_{i} \Delta t\right)-M_{i}^{n-1}+\frac{2 F^{n}}{w_{i}^{2}}\left(1-\cos \left(w_{i} \Delta t\right)\right),
$$

where the superscript indicates the number of time steps, $w_{i}$ $=c \pi \sqrt{\left(i_{x}^{2} / l_{x}^{2}+i_{y}^{2} / l_{y}^{2}+i_{z}^{2} / l_{z}^{2}\right)}, F^{n}$ is the DCT of force $f(\mathbf{x}, t)$ at $n$th time step, and $\Delta t$ is the size of the time step. Mode coefficients are then transformed back into pressure by an inverse DCT. This gives the pressure inside each rectangular partition. The pressure is propagated across neighboring partitions by performing interface handling using a sixth-order finite-difference stencil. To incorporate sound absorption at the partition boundaries, Perfectly Matched Layer (PML) absorbing boundary conditions are used. Currently, the ARD 
simulator can handle absorption and reflection of sound while ignoring transmission through objects. For more details, please refer to the original texts. ${ }^{14,33}$

The ARD technique is more efficient than the FDTD technique because of its larger grid spacing and time steps. The grid spacing for the ARD technique is $h=c /\left(\nu_{\max } s\right)$, where $\nu_{\max }$ is the maximum simulation frequency and $s=2.6$ is number of samples per wavelength. The ARD's simulation time step is restricted by the Courant-Friedrichs-Lewy (CFL) condition $\Delta t \leq h /(c \sqrt{3})$. In contrast, the FDTD technique requires a much higher value of samples per wavelength ( $s=10$ used in Taflove et al. ${ }^{36}$ or $s=20$ used in Albert and $\mathrm{Liu}^{13}$ ), resulting in much denser grid and smaller time steps. Therefore, the ARD technique is computationally more efficient and requires less memory than the FDTD technique. This efficiency enables the ARD technique to perform 3D wave-simulations on large, complex scenes at a higher simulation frequency than FDTD can, all on a desktop computer.

The ARD technique has few intrinsic limitations. Its primary limitation is its assumption that the speed of sound is spatially invariant. Sound speed can change spatially due to many factors, such as temperature gradient, humidity, or wind, and the ARD technique does not model the effect of these factors on sound propagation. Another limitation is that the atmospheric absorption is currently not modeled in the simulation. Also, the simulation does not model sound transmission through the objects (walls, buildings, etc). In a general scenario, these limitations can have an effect on the quality of the simulation results. However, in the present study, these limitations have negligible effect on the prediction quality. The sensors are placed at a height of $1.5 \mathrm{~m}$ and most of the energy recorded at these sensors is due to the acoustic propagation happening close to the ground $(<15 \mathrm{~m})$. For such small elevations, the temperature gradient of the atmosphere is negligible and therefore does not affect the speed of sound. The wind speed is also too low $(2-5 \mathrm{~m} / \mathrm{s})$ to have any significant effect on arrival times of the acoustic pulses. Also, atmospheric absorption can be safely ignored in this case, as the simulation frequency is less than $500 \mathrm{~Hz}$ and the propagation distances are on the order of hundreds of meters, for which the intrinsic absorption of the atmosphere is negligible. As for sound transmission, due to the very high impedance contrast between the air and the concrete buildings, very little acoustic energy can get transmitted through the buildings.

\section{B. Validation}

We provide validation results of the ARD technique on two benchmark test-cases: (a) spherical wave scattering by a rigid sphere, and (b) edge-diffraction from a right-angled rigid wall. In the first case, the acoustic wave equation has known analytical solution. ${ }^{37}$ The scene setup is as follows: A sphere of radius $a=1 \mathrm{~m}$, surrounded by air with speed of sound $343 \mathrm{~m} / \mathrm{s}$ and mean density of $1.21 \mathrm{~kg} / \mathrm{m}^{3}$, is centered at origin $(0,0,0)$. A spherical sound source (monopole source) is placed at position $(0,0,-3 \mathrm{~m})$. The spherical wave emitted by the source is scattered by the rigid sphere. The total field (incident + scattered field) is computed using the analytical solution of the wave equation at an angular distribution of listener positions situated at a distance of $1.5 \mathrm{~m}$. The analytical solutions are compared against the simulation results at different wave numbers $k$ as shown in Fig. 3. The results are plotted versus the polar angle $\theta$, where $\theta=180^{\circ}$ corresponds to the front end of sphere with respect to the incoming spherical wave. The comparisons between the analytical expressions and the ARD simulation results show very good agreement.

In the second case, we perform validation of the ARD technique by comparing it against the edge diffraction model proposed by Svensson et al. ${ }^{38}$ This model is an extension of Biot-Tolstoy-Medwin solution ${ }^{39}$ to finite edges. The scene setup is as follows: A right-angled rigid wall of dimension $8 \mathrm{~m} \times 12 \mathrm{~m}$ is considered, with the longer edge being the diffraction edge. Source and receiver are placed at symmetric positions with respect to the wall at $(-1.8 \mathrm{~m},-0.9 \mathrm{~m}$, $-6.0 \mathrm{~m})$ and $(0.9 \mathrm{~m}, 1.8 \mathrm{~m},-6.0 \mathrm{~m})$, respectively. The timeand frequency-domain responses are computed using the BTM finite-edge diffraction model and compared against the results of the ARD simulation. As shown in Fig. 4, the agreement between the two responses is very good.

\section{Simulation parameters}

The source function used to model the explosive blast signal for calculations in the ARD simulator is described in Liu and Albert (2006). ${ }^{11}$ Figure 5 shows the corresponding source function with peak pressure normalized to 1 .

In order to run 3D numerical simulation, a virtual 3D model of the scene is required. However, a detailed 3D model cannot be constructed due to the lack of availability of architectural blueprints or a laser-scanned point cloud of the site. Therefore, we construct a simplified 3D model of the scene using a 2D layout of the village, photographs, and the heights of corners and rooftops of buildings. This 3D model is an approximation to the actual geometry of the scene, since it lacks particular geometric details (peaked roofs, door/window locations, facade details, and extraneous geometry such as cars and a fountain). The dimensions of the simulation domain are $175 \mathrm{~m} \times 140 \mathrm{~m} \times 14 \mathrm{~m}$. The heights of the buildings are between 6-9 $\mathrm{m}$. Therefore, depending on the building, a vertical space of 5-8 $\mathrm{m}$ exists between the top of the roof and the top of the simulation domain (excluding PML), allowing correct simulation of rooftop diffraction. Figure 1(b) is a textured rendering of the $3 \mathrm{D}$ model. Based on the type of material present (e.g., concrete, grass, soil, etc.), we assign the appropriate absorption coefficients to the surfaces of the 3D model.

The ARD simulation was run with an acoustic wave velocity of $375 \mathrm{~ms}^{-1}$ and an air density of $1.2 \mathrm{~kg} \mathrm{~m}^{-3}$. The high value for the acoustic wave velocity comes from the propagation of the high-amplitude acoustic pulse generated by the $\mathrm{C} 4$ explosive used as the sound source. In the case of concrete, the acoustic wave velocity is $2950 \mathrm{~ms}^{-1}$ and density is $2300 \mathrm{~kg}$ $\mathrm{m}^{-3}$, which results in a reflection coefficient of 0.99 for concrete. These parameters correspond to the values used in Albert and Liu (2010) ${ }^{13}$ for the finite-difference simulation.

We run the ARD simulator to propagate the acoustic pulse from each given sound source position, one by one. We record the responses at the specified receiver positions and compare the results with the recorded measurement data. 

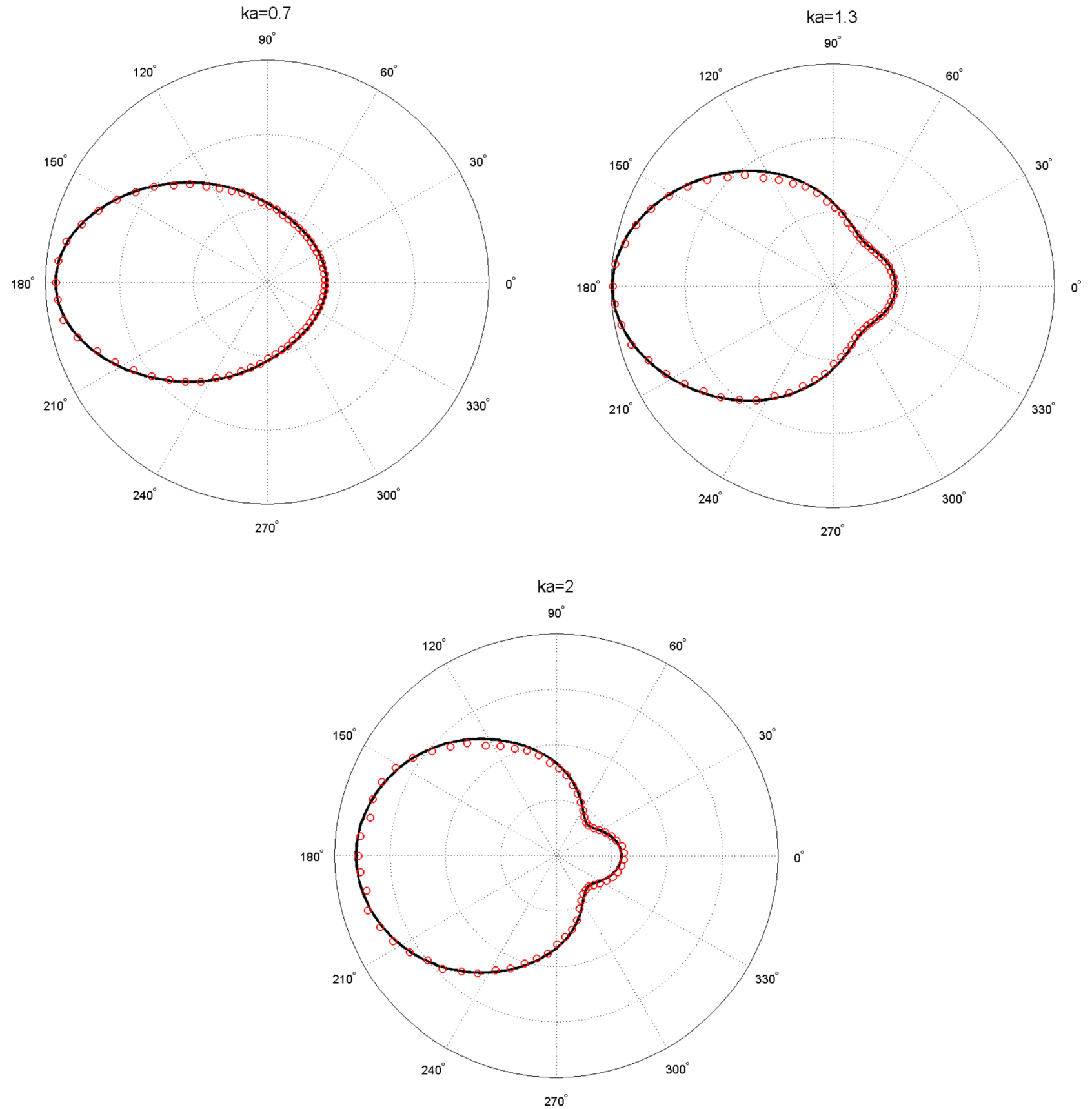

FIG. 3. (Color online) Validation of the ARD simulation results (dots) with analytical expressions (curves) for the scattering of a spherical wave by a rigid sphere. Normalized pressure is plotted in the radial axis. The radius of the sphere $a=1 \mathrm{~m}$ and wave numbers $k$ considered are $0.7 \mathrm{~m}^{-1}, 1.4 \mathrm{~m}^{-1}$, and $2 \mathrm{~m}^{-1}$, respectively.

\section{The 3D vs 2D wave simulation}

We discuss the advantages of the 3D wave simulator over the 2D wave simulator for acoustic pulse propagation. First, a 3D wave simulation incorporates propagation paths over the top of walls or buildings, as well as wave diffraction from the upper edges, both of which are completely ignored by a $2 \mathrm{D}$ simulation. Second, in $3 \mathrm{D}$ simulation, the sound reflection from the ground terrain is handled accurately for all frequencies. For a 2D simulation, the pressure is simply doubled to approximate ground reflections, which is accurate only for frequencies up to $600 \mathrm{~Hz}$, as discussed in Liu and Albert (2006). Last, the results of a 2D simulation must be renormalized by an additional factor of $1 / \sqrt{r}$ to account for
3D geometric spreading. This normalization is valid only for large $\mathrm{kr}$ (where $\mathrm{k}$ is the wave number and $\mathrm{r}$ is distance to the source). A 3D simulation requires no such normalization.

\section{RESULTS}

In this section, we compare the waveforms calculated by the ARD simulator with the measurements recorded in the village and with the waveforms calculated by the $2 \mathrm{D}$ FDTD simulation proposed by Albert and Liu (2010).

\section{A. Simulation}

The ARD simulations were run for the four source positions, SP1 to SP4, shown in Fig. 1(a). The parameters used 


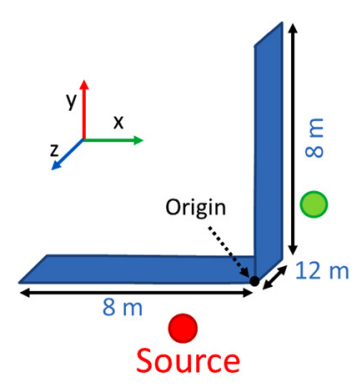

(a)
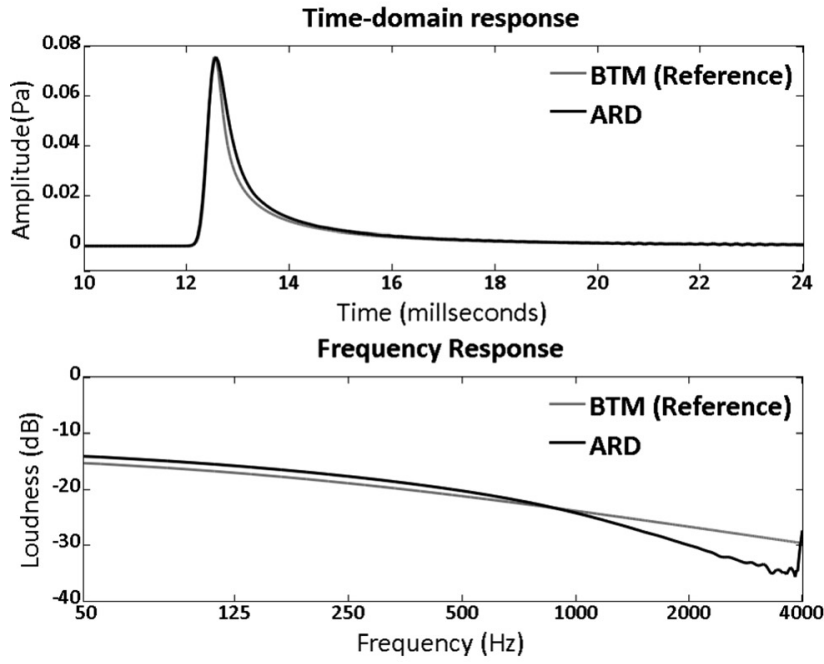

(b)

FIG. 4. (Color online) Time and frequency responses produced by the BiotTolstoy-Medwin diffraction model (reference) and the ARD simulation for a right-angled rigid wall. Grid spacing $h$ used in the ARD simulation is given by $h=c /\left(\nu_{\max } s\right)$ where $c$ is the speed of sound, $\nu_{\max }$ is the simulated frequency, and $s$ is samples per wavelength ( $s=2.6$ for ARD).

in the simulator are given in Table I. The total simulation time for each source was $20 \mathrm{~min}$ for the CPU-based ARD implementation ${ }^{14}$ and $1-2 \mathrm{~min}$ for the GPU-based ARD implementation. ${ }^{33}$ The CPU-based implementation is in $\mathrm{C}++$ and the GPU-based implementation uses NVIDIA's CUDA. These timing results were measured on a single core of a 4-

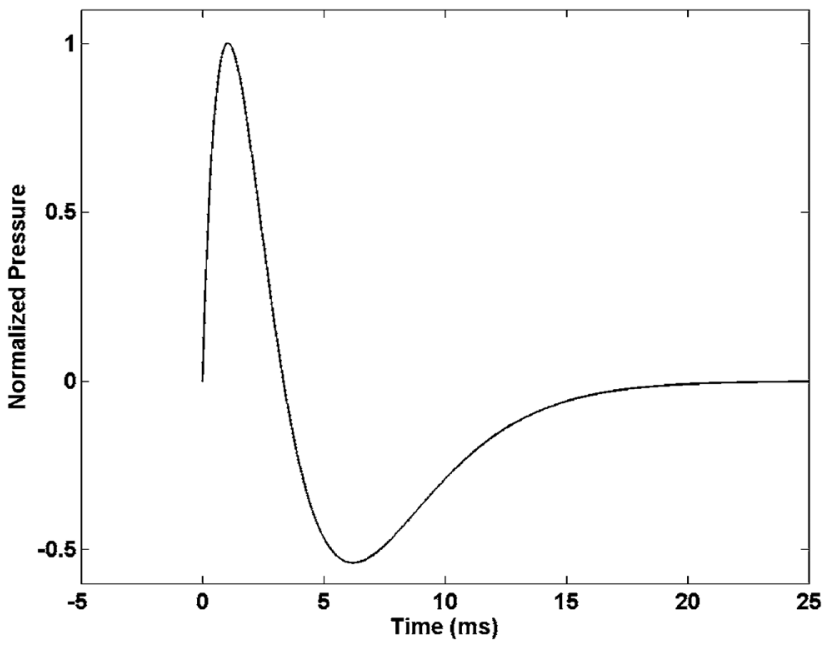

FIG. 5. The source pulse used for modeling the blast signal produced in the experiment as calculated from Eq. (5) (Liu and Albert, 2006) (Ref. 11).
TABLE I. Parameters for the ARD technique.

\begin{tabular}{lc}
\hline \hline Parameters & Values \\
\hline Simulation frequency & $450 \mathrm{~Hz}$ \\
Grid size & $175 \mathrm{~m} \times 140 \mathrm{~m} \times 14 \mathrm{~m}$ \\
Grid spacing & $0.31 \mathrm{~m}$ \\
\# Grid points & $11 \mathrm{million}$ \\
Time step size & $385 \mu \mathrm{s}$ \\
\# Time steps & 2000 \\
Simulation length & $0.77 \mathrm{~s}$ \\
\hline \hline
\end{tabular}

core $2.80 \mathrm{GHz}$ Xeon X5560 desktop machine with $4 \mathrm{~GB}$ of RAM and on a NVIDIA GeForce GTX 480 GPU with 448 CUDA cores and 1.5 GB memory.

The ARD responses were computed for four source positions, up to a maximum frequency of $450 \mathrm{~Hz}$. The measured waveforms were low-passed to $450 \mathrm{~Hz}$ for source positions 2 and 3 to compare with the calculated ARD waveforms. For source positions 1 and 4, both the ARD and the measured responses were low-passed to $200 \mathrm{~Hz}$, since the 2D FDTD waveforms are available only up to $200 \mathrm{~Hz}$. In this scene, the simulation frequency is less than $500 \mathrm{~Hz}$ and the propagation distances are on the order of hundreds of meters, for which the intrinsic absorption of the atmosphere is negligible. Therefore, we have ignored atmospheric absorption during the simulation.

Figure 6 shows the visualization of the time-domain ARD simulation at specified time steps. These visualizations show the propagation of wave fronts in the scene and how they are modified by the multiple reflections and diffractions from the buildings, and reflections from the ground. These can be helpful in guiding engineering modifications to the scene.

\section{B. Varying propagation speed}

In the training village scene considered in this study, the sound source used is a C4 explosive. The high-amplitude explosion caused by this source generates a varying soundspeed profile. Figure 5 in Albert and Liu (2010) shows the measured values: Over $400 \mathrm{~m} / \mathrm{s}$ a meter or two away and

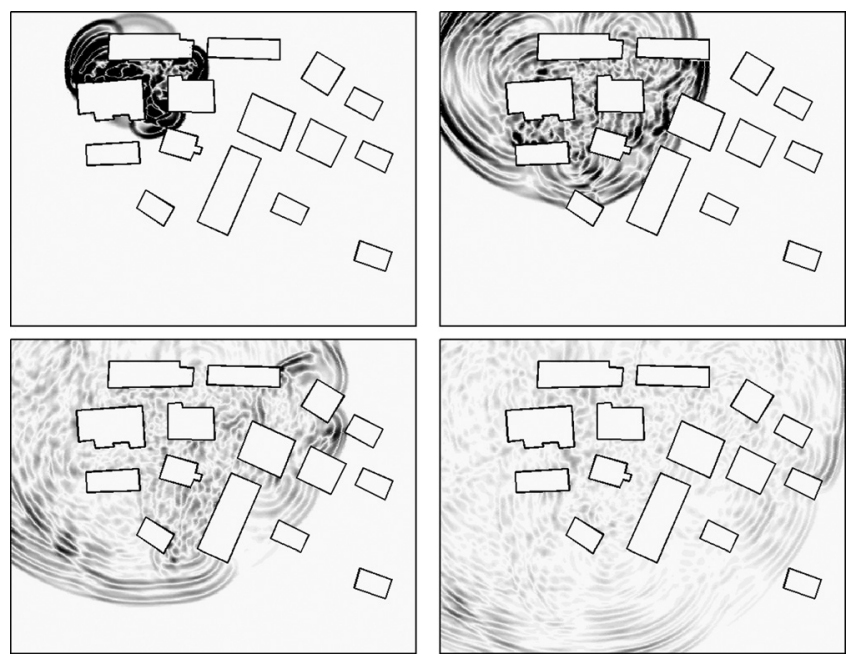

FIG. 6. Calculated acoustic response for the source position SP1 in the artificial village scene using the ARD technique. Simulated wavefields are shown at times $\mathrm{t}=75,150,225$, and $300 \mathrm{~ms}$. 
$375 \mathrm{~m} / \mathrm{s}$ at about $20 \mathrm{~m}$ distance. The speed varies with the amplitude of the traveling pulse, reducing with distance. To make things more complicated, the amplitude of a wave that diffracts around a building corner is reduced, and that diffracted wave travels slower than waves at the same propagation distance that are in line-of-sight paths. This generates a complicated wave-speed profile that is harder to model. The measured waveform's peak arrival times can differ from those of the calculated waveforms, which assume a constant speed of sound. The individual peaks can also get stretched due to decreasing wave speed in the measured waveforms. There are complicated changes in acoustic wave speed even for different waves at the same location.

As in the 2D finite difference simulation of Albert and Liu (2010), ${ }^{13}$ our 3D ARD simulation does not allow us to track the changes caused due to amplitude. To do that correctly, one would have to either follow the individual wavefronts or modify the code to explicitly include non-linear effects. Instead, we chose a constant wave speed to get the best waveform fits when compared with the measured data. Also, similar to Albert and Liu (2010), ${ }^{13}$ we time-shift the response of the linear simulation to align the first arrival peak with the measured waveform. In addition to that, we try to account for these kinematic errors due to the varying sound speed with the "robust" error metric discussed later.

\section{Error metrics}

\section{Basic error metric}

In order to perform a quantitative comparison of the measured and the calculated waveforms, two types of error metrics are used in the comparison: The spectrogram difference metric and the average $\mathrm{dB}$ error metric. The spectrogram difference metric $(\mathrm{SDM})$ is computed on the spectrograms SPEC of the time-domain pressure signals $\{a\}_{i=1}^{N}$ and $\{b\}_{i=1}^{N}$ :

$$
\operatorname{SDM}(a, b)=\frac{\sum_{j=1}^{M} \sum_{k=1}^{T}\left\|\operatorname{SPEC}(a)_{j k}-\operatorname{SPEC}(b)_{j k}\right\|^{2}}{\sum_{j=1}^{M} \sum_{k=1}^{T}\left\|\operatorname{SPEC}(a)_{j k}\right\|^{2}}
$$

where $M=\{N / 2$ if $N$ is even or $N / 2+1$ if odd $\}$ and $T$ is the number of time segments in the spectrogram.

The average $\mathrm{dB}$ error metric (ADM) is computed on the pressure signals in the $\mathrm{dB}$ scale $\{d B(a)\}_{i=1}^{N}$ and $\{d B(b)\}_{i=1}^{N}$ as follows:

$$
A D M(a, b)=\sum_{i=1}^{N}\left\|d B(a)_{i}-d B(b)_{i}\right\| / N
$$

\section{Robust error metric}

The error metrics defined above are very sensitive to the time of arrival in the waveforms. In scenarios where the speed of sound is constant, these metrics perform well. However, in this case, where the speed of sound varies as discussed in Sec.
$\mathrm{VB}$, the arrival times can be off by a few milliseconds. Though this small time-shift might not cause a big difference in individual waveform characteristics, such as shape, frequency content, etc., it can generate a large error with these error metrics. Although it would appear that regular cross correlation could remedy this situation, it will match the peak arrival but will misalign the rest of the measured waveform.

We propose changes to the above error metrics that will make them more robust to the small time-shifts and peak stretching caused by varying sound speeds. Our proposed change is based on the idea of Dynamic Time Warping (DTW), which is a standard tool in signal-processing community to handle non-linear transformations in the time axis. In the DTW technique, the two input time signals are allowed to shift, stretch, or contract in a limited manner to generate the optimal match between the signals. This technique has been widely used in the areas of speech processing, ${ }^{40}$ acoustics, ${ }^{41}$ bioinformatics, ${ }^{42}$ and medicine. ${ }^{43}$

In our proposed solution, we align the first arrival peaks of the calculated and measured waveforms. This removes any time-shift before the arrival of the first peak. Next, we take these signals and apply the DTW technique to align the remaining part, thus taking into account small time shifts and stretching. Finally, the above error metrics are applied to these aligned signals to give a quantitative measure of the error; we define at the same time the confidence we have in the resulting measure. The confidence measure is based on the intuition that the less warping required to align the signals, the more confidence we have in the similarity of the signals.

$$
\text { Confidence } \left.=\left(1-d_{w} / l_{o}\right) \times 100 \text { (in percent }\right)
$$

where $d_{w}=\left|l_{w}-l_{o}\right|$ is the difference in the length of the warped signal $l_{w}$ and the original signal $l_{o}$. For all results shown in this paper, we allow a warping length change of only $5 \%-10 \%$, resulting in a confidence measure of $90 \%-95 \%$.

\section{Comparison with measurements in time domain}

In this section, we compare the waveforms calculated using the ARD simulation with the measured waveforms for different source and receiver positions. Note that waveform modeling requires a strong agreement between the amplitudes and phases of the calculated and measured waveforms, making it a stringent test for any acoustic pulse propagation technique.

Figure 7 compares the ARD waveforms and the measurements for the source SP2 and the receiver positions. The upper traces in each panel correspond to the calculated ARD waveforms, and the lower traces correspond to the measurements. The source is located to the left of the narrow street canyon formed between buildings A and E [see Fig. 1(a)]. For all the line-of-sight (LOS) positions (R01 thru R06), we get an excellent match between the calculated and measured waveforms for the direct sound (first arrival) and the subsequent reflections. The main characteristic of LOS responses is the strong first arrival which dominates the signal, followed by (comparatively) weaker reflections. For receivers very close to the source (R01 thru R04), the high amplitude of direct sound completely dominates the later reflections, whereas for receivers far away (R05, R06), the reflections have considerable energy. 

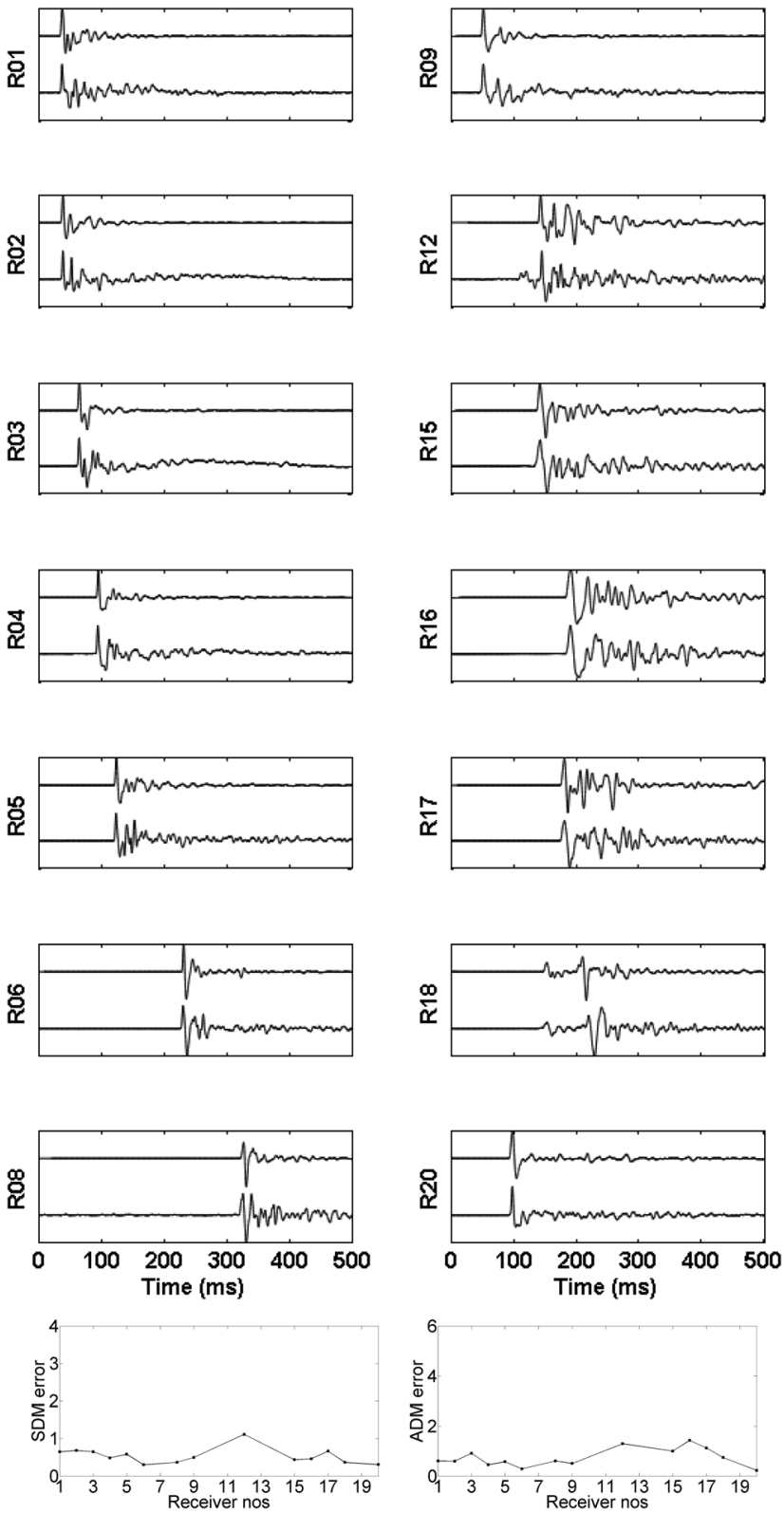

FIG. 7. Waveforms calculated by the 3D ARD simulator (upper) and the measured data (lower) in the artificial village for source position 2 at 14 receiver positions. All the waveforms have been individually normalized and low-passed to the maximum frequency of $450 \mathrm{~Hz}$. The error between the 3D ARD simulation and measurement has been calculated using the DTWbased SDM and ADM metric.

For non-line-of-sight (NLOS) positions, the waveform characteristic is position dependent and more complex. In the case of receiver R20, the propagation paths mainly consist of diffraction around and from the top of building E. For receiver R08, the diffracted first arrival is followed by highorder reflections from buildings $\mathrm{C}, \mathrm{H}, \mathrm{D}$, and I. In the case of receiver R15, diffracted arrivals around and from the top of buildings $\mathrm{A}$ and $\mathrm{B}$ are followed by high-order reflections and diffractions from A, F, and B. Similarly, for R09, the first arrival corresponds to diffraction from the rooftop of building A followed by high-order reflections and diffractions from buildings $\mathrm{A}$ and E. For receivers R17 and R18 the first arrival is via diffraction, followed by multiple reflections trapped between buildings $F$ and G. In the case of R17, the diffraction angle is $10^{\circ}-15^{\circ}$, resulting in a high amplitude diffraction peak; in R18, by contrast, the diffraction angle is $90^{\circ}$, which results in a low amplitude diffraction peak.

As can be seen in Fig. 7, the calculated waveforms incorporate all the features and match with the measured waveforms to a high degree of accuracy. The biggest mismatch between the waveforms is for sensor R12, which is a NLOS position around the corner of the building A on Main Street. In this case, the ground floor room at the corner had two open windows facing Main Street and one open window around the corner, between Main Street and the sensor at $\mathrm{R} 12$. This resulted in a shorter path through that room to the sensor for sound coming from source positions SP2. This is probably what can be seen before the large arrival, which presumably diffracted around the building corner itself. The open windows had no glass at all and were about $2 \mathrm{ft} \times 4 \mathrm{ft}$ in area, so this small size (compared to a wavelength of about $7 \mathrm{~m}$ at the source) would reduce the amount of energy traveling on that shorter "indoor" path. Some of the additional high frequency arrivals later on in the measured waveform may be caused by reverberation inside that room. Because of lack of availability of window-position data, these were not included in the virtual 3D model constructed for the ARD simulation. Therefore, these early arrivals are not modeled in the simulation results. The same behavior is observed at receiver R12 for source positions SP1 and SP3.

In terms of the basic error metric (not shown in the figure), the highest value for this source simulation occurs at receiver R18 (basic SDM error =1.67) due to a decrease in the wave speed after the first diffraction from building F. This causes subsequent strong reflection from the opposite building $\mathrm{G}$ to arrive much later in time than the calculated waveform (which assumes a constant sound speed $375 \mathrm{~ms}^{-1}$ ). This time stretching cannot be modeled by a constant time shift applied at the beginning of the signal. Thus, the measured waveforms for R18 appear to be the stretched equivalents of the calculated waveforms; this results in a higher error using the basic metric. The DTW-based robust error metric takes this stretching into account, correctly predicting a low error as shown in the figure. Similar behavior can be observed for receivers R16 and R17.

In Fig. 8, we perform the same comparison between calculated and measured waveforms for the source position SP3. All the LOS positions (R01 to R06, and R20) exhibit excellent matches between the calculated and measured waveforms. For NLOS positions involving first-order diffraction (i.e., R09), the calculated responses incorporate diffraction paths from both around and on top of the buildings to generate the correct waveform. For R08, the first diffraction arrival is weaker than the later reflection; this behavior is correctly modeled by the calculated ARD response. As described before, the measured waveforms at receivers R16, $\mathrm{R} 17, \mathrm{R} 18$ get stretched in time by the variable acoustic speed; this stretching results in high error with the unwarped metric and low error with DTW-based metric. Aside from sensor R12, the biggest mismatch between the waveforms is for receiver R15, for which the calculated waveform shows two distinct peaks, as compared to only one peak for the measured waveform. One possible explanation is that the 

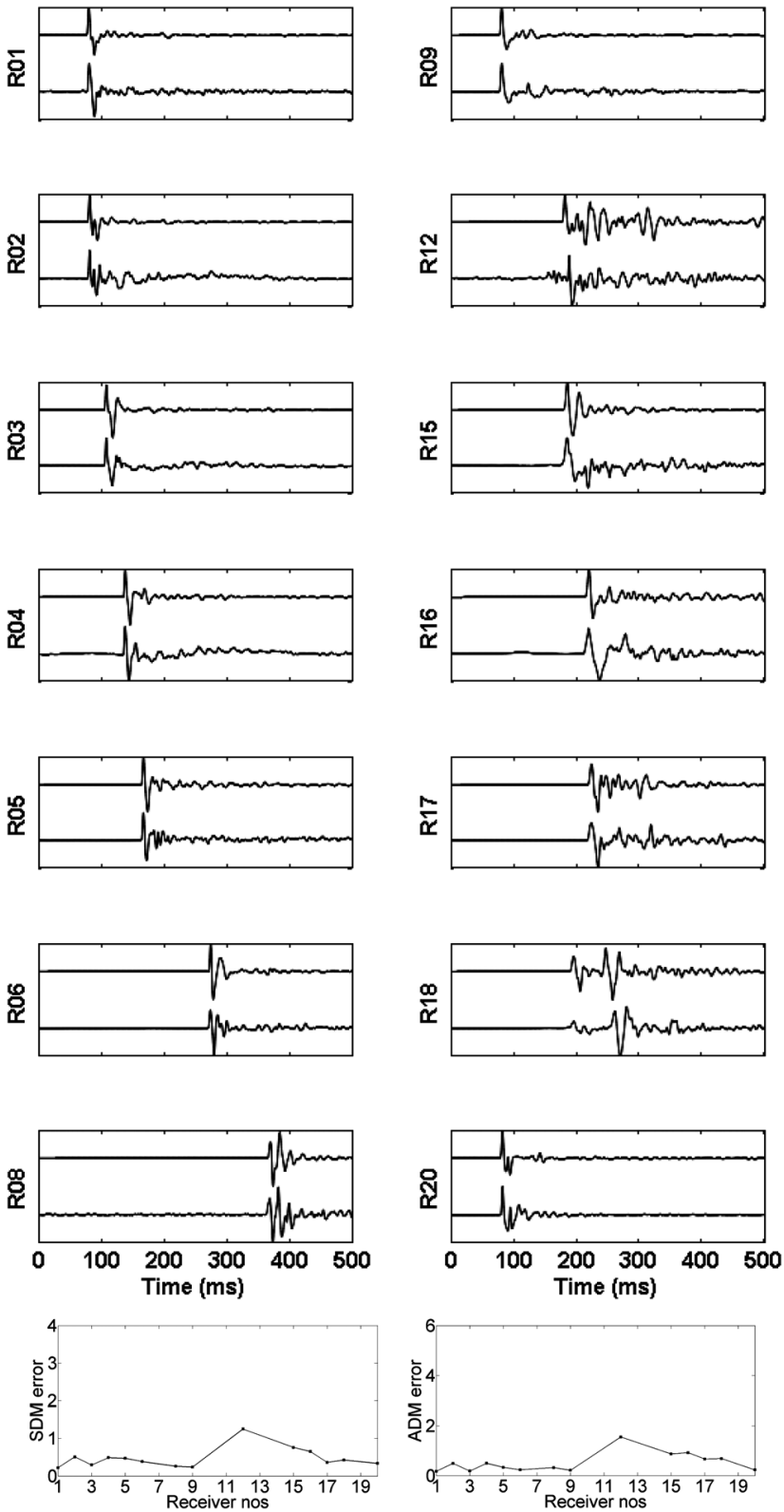

FIG. 8. Waveforms calculated by the 3D ARD simulator (upper) and the measured data (lower) in the artificial village for source position 3 at 14 receiver positions. All the responses have been individually normalized and low-passed to the maximum frequency of $450 \mathrm{~Hz}$. The error between the 3D ARD simulation and measurement has been calculated using the DTWbased SDM and ADM metric.

arrival times between the two diffraction peaks shown in the calculated response is smaller in the real scene due to varying speed of sound, resulting in a constructive interference and peak merging in the measured waveform.

\section{E. Comparison with 2D FDTD}

In this section, we compare the calculated ARD and FDTD waveforms to the measured waveforms for two source positions, SP1 and SP4. The ARD waveforms are calculated by running a 3D simulation on the 3D model. The FDTD waveforms are calculated by running a 2D finite-difference simulation on a 2D grid as described in Liu and Albert (2006). ${ }^{11}$

Figure 9 shows the calculated and measured waveforms for the source position SP1 and its receiver positions. The
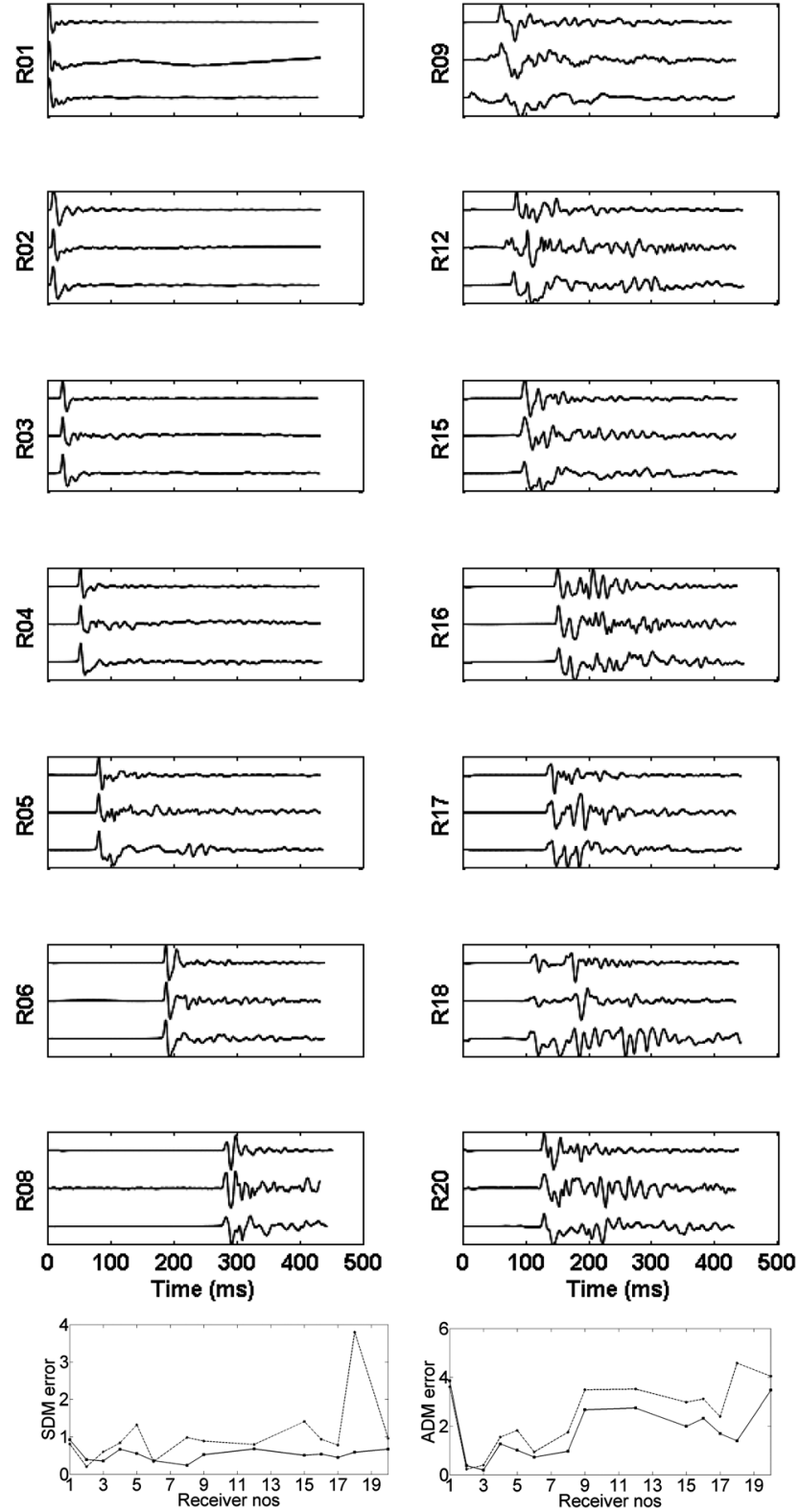

FIG. 9. Waveforms calculated by the 3D ARD simulations (upper), 2D FDTD simulations (lower), and the measurement data (middle) in the artificial village for source position 1 at 14 receiver positions. All the responses have been individually normalized and low-passed to the maximum frequency of $200 \mathrm{~Hz}$. The error between the 2D FDTD simulation and measurement (dashed line) and 3D ARD simulation and measurement (solid line) has been calculated using the DTW-based SDM and ADM metric.

upper trace in each panel correspond to the calculated ARD response, the middle trace to the measured waveform, and the lower trace to the calculated FDTD response. At LOS positions (R01 to R06), the dominant propagation happens in the $\mathrm{XY}$ plane containing the sources and receivers. Therefore, the waveforms calculated using the 3D ARD simulation and the 2D FDTD simulation match equally well to the measured waveforms. The main difference between a fully $3 \mathrm{D}$ and a $2 \mathrm{D}$ simulation arises in cases where the sound waves diffract over the rooftops of the buildings, resulting in shorter propagation paths and higher energy (as illustrated in Fig. 10). For receiver R09, the diffraction path from the top of building $\mathrm{A}$ is the shortest path and corresponds to the first 


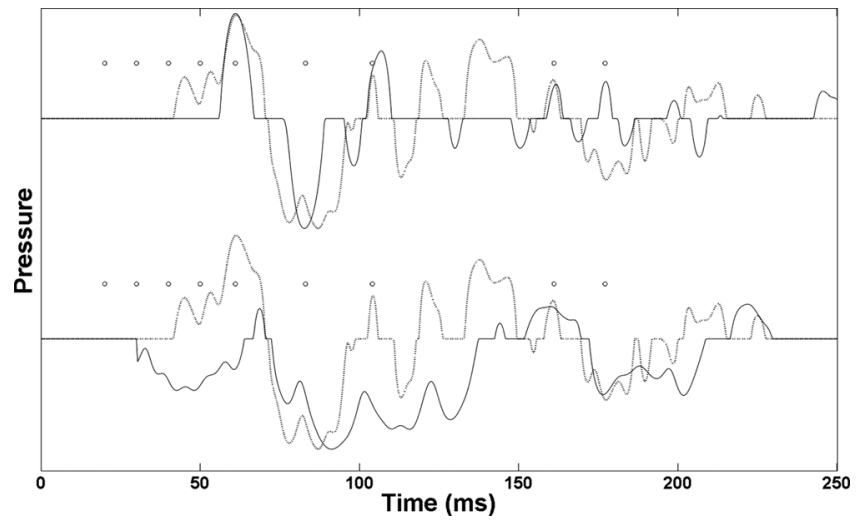

FIG. 10. Comparison between the calculated and measured waveforms for the source position SP1 and receiver R09 behind building A. The upper trace (solid line) corresponds to the 3D ARD waveform; the lower trace (solid line) corresponds to the 2D FDTD waveform. The measured waveform is drawn as dotted line. Note that the 2D FDTD simulation cannot model the diffraction path from the building's rooftop resulting in the missing first arrival at $60 \mathrm{~ms}$.

arrival. This shortest path is modeled correctly by the 3D ARD simulation. In the case of receiver R20, the secondary arrival peak corresponds to the rooftop diffraction path, which is missing in the $2 \mathrm{D}$ simulated waveform. For receiver $\mathrm{R} 15$, the energy from rooftop diffraction paths is missing from the 2D simulation but not from the 3D simulation. Therefore, for these NLOS cases, the 3D simulated waveforms match far better with the measured waveforms than the waveforms from the 2D simulation.

In Fig. 11, we do a similar comparison for source position SP4. This source is positioned outside the main village compound, and most of the receiver positions are NLOS. The only LOS position is receiver R20, where both the calculated waveforms match well with the measured waveforms. For NLOS positions, the measured waveforms are again stretched as compared to the calculated waveforms, as discussed before. The speed of sound reduces significantly after diffraction, resulting in high-order propagation peaks to arrive later in the measured waveforms than in ARD and FDTD waveforms (which assume constant speed of sound). In the case of receivers R04 to R06 and R15 to R18, the correct modeling of rooftop diffraction with a $3 \mathrm{D}$ simulation (ARD) results in a better match with the measured waveforms. The 2D FDTD simulation ignores these paths, resulting in a lower first-arrival energy than in the measured data. The measurement data for receiver position R1 is not available for the source position SP4. As described in Albert and Liu (2010), this location was still fitted with the high-pressure blast sensor from the previous measurement, when it was measuring the pressure from the nearby explosive charge at SP1. This high-pressure sensor was unable to detect the lowpressure waveform produced by the distant source SP4.

\section{F. Comparison with measurements in spatial domain}

One of the primary advantages of a time-domain wave simulation (FDTD or ARD) is the ability to save snapshots of the pressure field at any time step in the simulation. These snapshots can be assembled into a movie to elucidate wave-
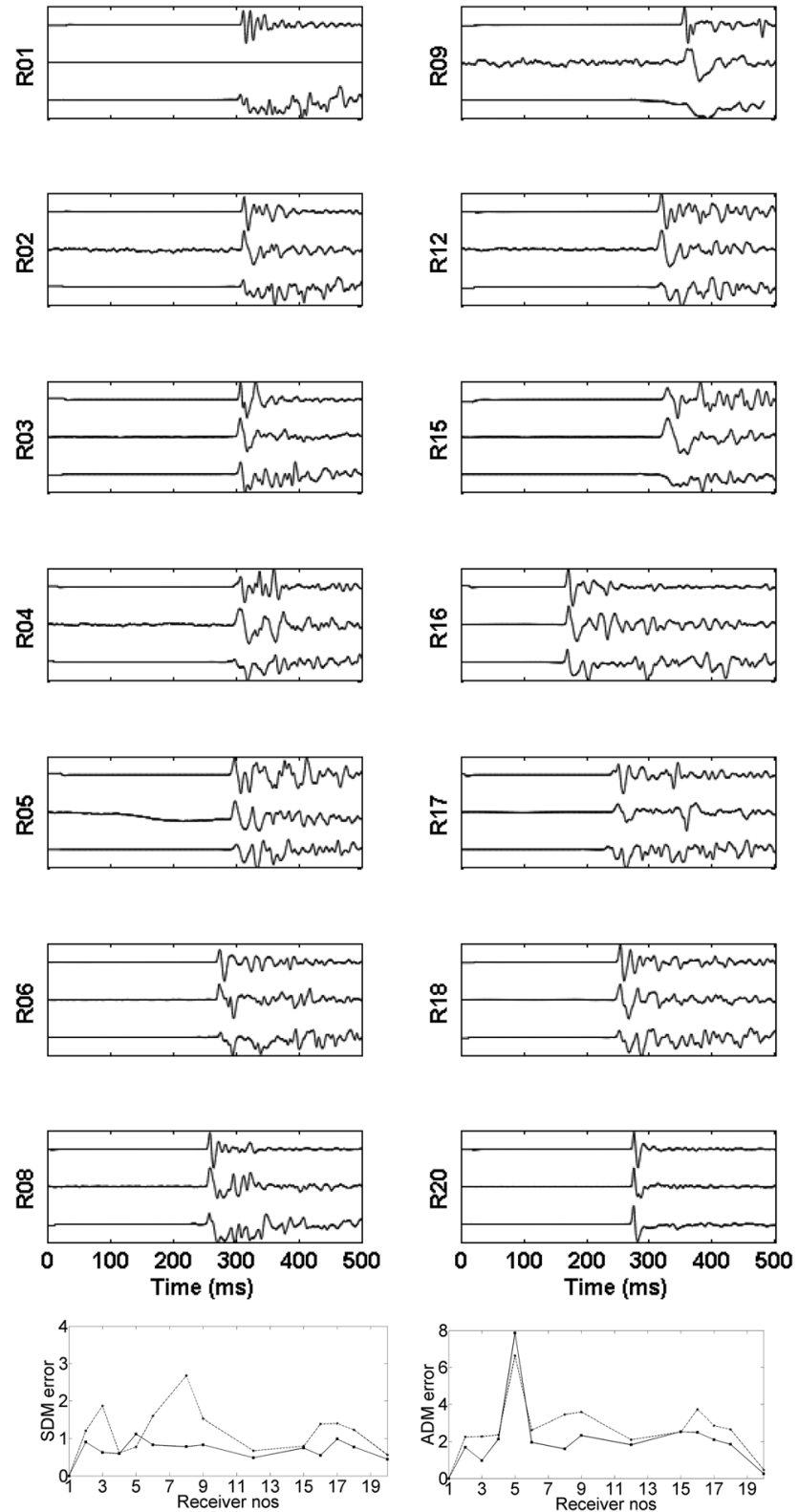

FIG. 11. Waveforms calculated by the 3D ARD simulations (upper), 2D FDTD simulations (lower), and the measurement data (middle) in the artificial village for source position 4 at 14 receiver positions. All the responses have been individually normalized and low-passed to the maximum frequency of $200 \mathrm{~Hz}$. The error between the 2D FDTD simulation and measurement (dashed line) and 3D ARD simulation and measurement (solid line) has been calculated using the DTW-based SDM and ADM metric.

field evolution in time as the acoustic pulse travels through the environment. This movie can serve as a useful tool for studying in detail the complex wave-interactions involved in the acoustic pulse propagation.

As an example, wave-field snapshots validate the presence of rooftop diffraction paths in both measured and 3D ARD waveforms for sensor R09 and source SP1 (see Fig. 12). In Fig. 10 (upper trace), the first arrival for $3 D$ ARD and measured waveforms happens at $t=61 \mathrm{~ms}$. The wave-field snapshot in Fig. 12 at $t=61 \mathrm{~ms}$ shows that the corresponding propagation path is a rooftop diffraction path from the top of building $\mathrm{A}$, followed by a diffraction from the side of the building at $t=69 \mathrm{~ms}$. The 2D FDTD simulation cannot 


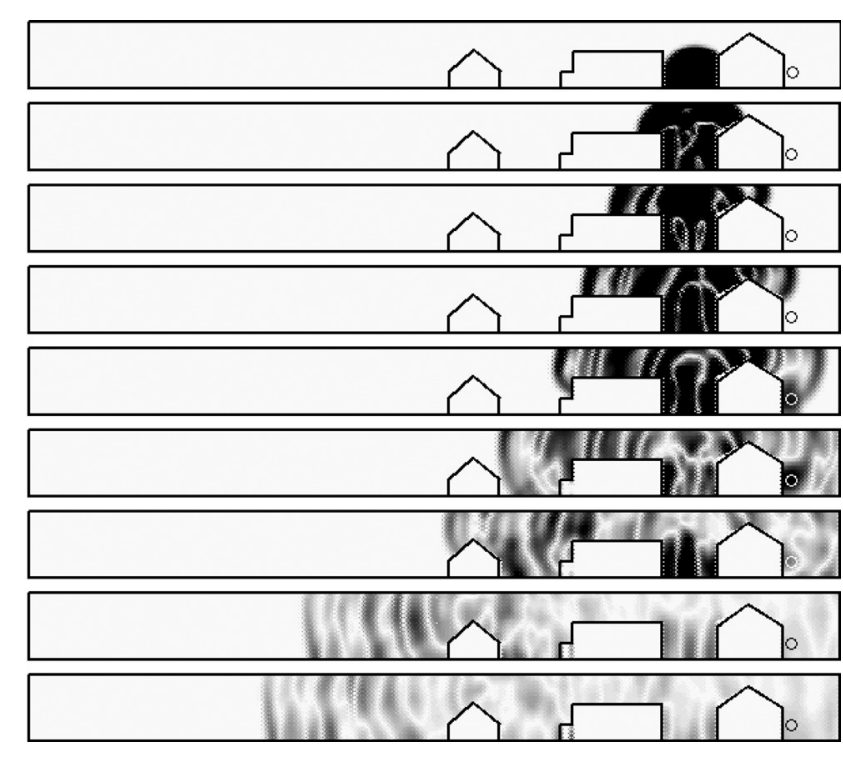

FIG. 12. Rooftop diffraction. Calculated acoustic response for the source position SP1 in the artificial village scene using the ARD technique. Receiver position R09 is marked by a circle on the side of the first building (from right). Simulated wavefields are shown at times $\mathrm{t}=20,30,40,50,61$, 83, 104, 161, and $177 \mathrm{~ms}$ corresponding to marked points in Fig. 10.

model 3D rooftop propagation paths and misses the energy corresponding to this path. Late arrivals in the waveform correspond to high-order reflections between building A and E that get diffracted around the side or the rooftop of building A before reaching the sensor. These correspond to peaks at $t=83,104$, and $177 \mathrm{~ms}$, which are marked by circles on top the waveforms in Fig. 10. These peaks are correctly modeled by the 3D ARD simulation, as shown by the wavefield snapshots for these times in Fig. 12. The wave-field snapshots and movies can thus provide a more thorough understanding of acoustic pulse propagation.

Figure 13 shows the variation of error with distance between the source-receiver positions. The error seems to be independent of the source-receiver distance. Note that error values are much higher for source position SP4 than others. This is due to the presence of more NLOS positions in SP4, which typically have more complex propagation characteristics than LOS positions. The top three sensors with consistently high errors across all sources are R12, R16, and R15. As discussed before, R12 has high errors due to open windows in building A, resulting in shorter propagation paths

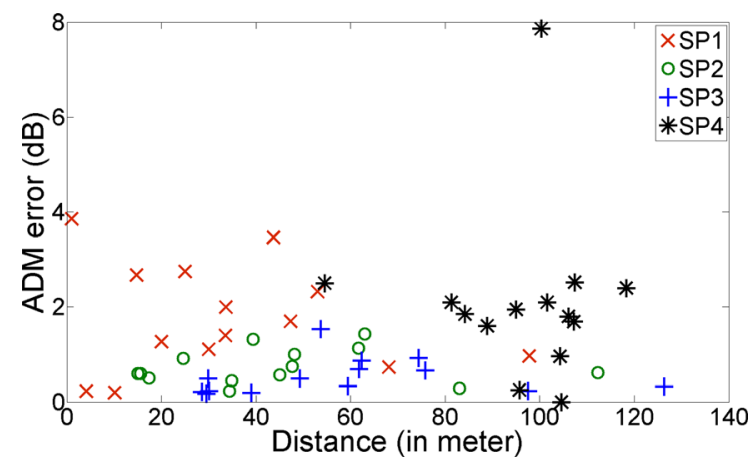

FIG. 13. (Color online) Variation of error with distance between the sourcereceiver positions. that are not modeled in the simulation. As for sensor R16 and R15, these are NLOS positions for all sources, which typically have higher errors.

\section{CONCLUSION, LIMITATIONS, AND FUTURE WORK}

In this paper, acoustic pulse propagation results are presented for a large urban environment in three dimensions. The results of the 3D simulation provide better agreement with the measured data than the 2D simulation, especially in cases where rooftop diffraction is involved. This technique enables acoustic propagation in a large three-dimensional scene with a broad frequency range on a desktop computer.

In future, we would like to explore the acoustic pulse propagation in three dimensions in the $\mathrm{kHz}$ range. This would require a very accurate geometric description of the scene (submeter accuracy) and parallelization of the ARD technique on a computer cluster. Second, we plan to investigate the benefit of a full 3D simulation for time reversal processing ${ }^{5}$ to compute the source location given the recorded waveforms at sensor positions. Last, we also plan to study the transmission of sound through buildings to determine the noise levels inside the buildings from exterior sources.

\section{ACKNOWLEDGMENTS}

This research was supported in part by the Link Foundation Fellowship in Advanced Simulation and Training, ARO Contracts W911NF-10-1-0506, W911NF-121-0430, W911NF-13-C-0037, and the National Science Foundation (NSF awards 0917040, 1320644). Additional funding for this research was provided by the U.S. Army Engineer Research and Development Center's Geospatial Research and Engineering business area.

${ }^{1}$ J. Kang, Urban Sound Environment (Taylor \& Francis, New York, 2006), $304 \mathrm{pp}$.

${ }^{2}$ D. May and N. Osman, "Highway noise barriers: New shapes," J. Sound Vib. 71, 73-101 (1980).

${ }^{3}$ D. Hothersall, S. Chandler-Wilde, and M. Hajmirzae, "Efficiency of single noise barriers," J. Sound Vib. 146, 303-322 (1991).

${ }^{4}$ A. Ledeczi, P. Volgyesi, M. Maroti, G. Simon, G. Balogh, A. Nadas, B. Kusy, S. Dora, and G. Pap, "Multiple simultaneous acoustic source localization in urban terrain," in Fourth International Symposium on Information Processing in Sensor Networks (2005), pp. 491-496.

${ }^{5}$ D. G. Albert, L. Liu, and M. L. Moran, "Time reversal processing for source location in an urban environment," J. Acoust. Soc. Am. 118, 616-619 (2005).

${ }^{6}$ F. M. Wiener, C. I. Malme, and C. M. Gogos, "Sound propagation in urban areas," J. Acoust. Soc. Am. 37, 738-747 (1965).

${ }^{7}$ R. Bullen and F. Fricke, "Sound propagation in a street," J. Sound Vib. 46, 33-42 (1976).

${ }^{8}$ K. K. Iu and K. M. Li, "The propagation of sound in narrow street canyons," J. Acoust. Soc. Am. 112, 537-550 (2002).

${ }^{9} \mathrm{~K}$. Heutschi, "A simple method to evaluate the increase of traffic noise emission level due to buildings, for a long straight street," Appl. Acoust. 44, 259-274 (1995).

${ }^{10} \mathrm{~J}$. Kang, "Sound propagation in interconnected urban streets: A parametric study," Environ. Plann. B 28, 281-294 (2001).

${ }^{11}$ L. Liu and D. G. Albert, "Acoustic pulse propagation near a right-angle wall,” J. Acoust. Soc. Am. 119, 2073-2083 (2006).

${ }^{12}$ W. C. K. Alberts, I. I. J. M. Noble, and M. A. Coleman, "Sound propagation in the vicinity of an isolated building: An experimental investigation," J. Acoust. Soc. Am. 124, 733-742 (2008).

${ }^{13}$ D. G. Albert and L. Liu, "The effect of buildings on acoustic pulse propagation in an urban environment," J. Acoust. Soc. Am. 127, 1335-1346 (2010). 
${ }^{14}$ N. Raghuvanshi, R. Narain, and M. C. Lin, "Efficient and accurate sound propagation using adaptive rectangular decomposition," IEEE Trans. Visual. Comput. Graphics 15, 789-801 (2009).

${ }^{15}$ A. Pierce, Acoustics: An Introduction to Its Physical Principles and Applications (Acoustical Society of America, New York, 1989), 678 pp.

${ }^{16}$ K. P. Lee and H. G. Davies, "Nomogram for estimating noise propagation in urban areas," J. Acoust. Soc. Am. 57, 1477-1480 (1975).

${ }^{17}$ A. Garcia and L. Faus, "Statistical analysis of noise levels in urban areas," Appl. Acoust. 34, 227-247 (1991).

${ }^{18}$ J. Picaut, T. Le Pollès, P. L'Hermite, and V. Gary, "Experimental study of sound propagation in a street," Appl. Acoust. 66, 149-173 (2005).

${ }^{19}$ J. Kang, "Sound propagation in street canyons: Comparison between diffusely and geometrically reflecting boundaries," J. Acoust. Soc. Am. 107, 1394-1404 (2000).

${ }^{20} \mathrm{D}$. Botteldooren, "Acoustical finite-difference time-domain simulation in a quasi-Cartesian grid,” J. Acoust. Soc. Am. 95, 2313-2319 (1994).

${ }^{21}$ L. L. Thompson, "A review of finite-element methods for time-harmonic acoustics," J. Acoust. Soc. Am. 119, 1315-1330 (2006).

${ }^{22}$ C. A. Brebbia, Boundary Element Methods in Acoustics (Springer, New York, 1991), $312 \mathrm{pp}$.

${ }^{23} \mathrm{M}$. Ochmann, "The source simulation technique for acoustic radiation problems," Acta Acust. Acust. 81, 512-527 (1995).

${ }^{24}$ J. P. Boyd, Chebyshev and Fourier Spectral Methods (Dover Publications, New York, 2001), 668 pp.

${ }^{25}$ E. Premat and Y. Gabillet, "A new boundary-element method for predicting outdoor sound propagation and application to the case of a sound barrier in the presence of downward refraction," J. Acoust. Soc. Am. 108, 2775-2783 (2000).

${ }^{26} \mathrm{~S}$. Chandler-Wilde, “The boundary element method in outdoor noise propagation," Proc. Inst. Acoust. 90, 27-50 (1997).

${ }^{27}$ M. Ogren and W. Kropp, "Road traffic noise propagation between two dimensional city canyons using an equivalent sources approach," Acta Acust. Acust. 90, 293-300 (2004).

${ }^{28}$ T. Van Renterghem, E. Salomons, and D. Botteldooren, "Parameter study of sound propagation between city canyons with a coupled FDTD-PE model," Appl. Acoust. 67, 487-510 (2006).

${ }^{29}$ N. C. Ovenden, S. R. Shaffer, and H. J. S. Fernando, "Impact of meteorological conditions on noise propagation from freeway corridors," J. Acoust. Soc. Am. 126, 25-35 (2009).

${ }^{30}$ M. Hornikx, R. Waxler, and J. Forssen, "The extended Fourier pseudospectral timedomain method for atmospheric sound propagation," J. Acoust. Soc. Am. 128, 1632-1646 (2010).
${ }^{31}$ S. A. Ketcham, D. K. Wilson, M. W. Parker, and H. H. Cudney, "Signal fading curves from computed urban acoustic wave fields," Proc. SPIE 6963, 1-8 (2008).

${ }^{32}$ T. L. Pollès, J. Picaut, M. Bérengier, and C. Bardos, "Sound field modeling in a street canyon with partially diffusely reflecting boundaries by the transport theory," J. Acoust. Soc. Am. 116, 2969-2983 (2004).

${ }^{33}$ R. Mehra, N. Raghuvanshi, L. Savioja, M. C. Lin, and D. Manocha, "An efficient gpu-based time domain solver for the acoustic wave equation," Appl. Acoust. 73, 83-94 (2012).

${ }^{34}$ D. K. Wilson, "Outdoor sound propagation: Recent modeling developments and applications to noise control," in Internoise 2011 Proceedings, K-4 (2011), paper 439998.

${ }^{35}$ N. Raghuvanshi, R. Mehra, D. Manocha, and M. C. Lin, “Adaptive rectangular decomposition: A spectral, domain-decomposition approach for fast wave solution on complex scenes," J. Acoust. Soc. Am. 132, 1890-1890 (2012).

${ }^{36}$ A. Taflove and S. C. Hagness, Computational Electrodynamics: The Finite-Difference Time-Domain Method (Artech House Publishers, London, UK), 1006 pp.

${ }^{37}$ T. Hasegawa, M. Ochi, and K. Matsuzawa, "Acoustic radiation pressure on a rigid sphere in a spherical wave field," J. Acoust. Soc. Am. 67, 770-773 (1980).

${ }^{38}$ U. P. Svensson, R. I. Fred, and J. Vanderkooy, "An analytic secondary source model of edge diffraction impulse responses," J. Acoust. Soc. Am. 106, 2331-2344 (1999).

${ }^{39}$ H. Medwin, E. Childs, and G. M. Jebsen, "Impulse studies of double diffraction: A discrete Huygens interpretation," J. Acoust. Soc. Am. 72, 1005-1013 (1982).

${ }^{40}$ H. Sakoe and S. Chiba, "Dynamic programming algorithm optimization for spoken word recognition," IEEE Trans. Acoust. Speech Signal Processing 26, 43-49 (1978).

${ }^{41}$ C. Masterson, G. Kearney, and F. Boland, "Acoustic impulse response interpolation for multichannel systems using dynamic time warping," in 35th AES Conference on Audio for Games (Audio Engineering Society, New York, 2002), p. 34.

${ }^{42}$ J. Aach and G. M. Church, "Aligning gene expression time series with time warping algorithms," Bioinformatics 17, 495-508 (2001).

${ }^{43}$ E. Caiani, A. Porta, G. Baselli, M. Turiel, S. Muzzupappa, F. Pieruzzi, C. Crema, A. Malliani, and S. Cerutti, "Warped-average template technique to track on a cycleby-cycle basis the cardiac filling phases on left ventricular volume," in Computers in Cardiology (IEEE, New York, 1998), pp. 73-76. 